\title{
Lymphoepithelioma of Larynx: Case Report
}

\author{
(D) Belgin Tutar ${ }^{1}$, (D) Fatih Akgün ${ }^{1}$, (D) İsmail Abdullahi Ahmed ${ }^{1}$, (D) Güler Berkiten ${ }^{1}$, (D) Ziya Saltürk ${ }^{1}$, (D) Yavuz Uyar ${ }^{1}$, \\ (D) Pınar Özay Nayır² \\ 1 University of Health Sciences Turkey, Prof. Dr. Cemil Taş̧̧ığlu City Hospital, Clinic of Otorhinolaryngology, istanbul, Turkey \\ 2University of Health Sciences Turkey, Prof. Dr. Cemil Taşçığlu City Hospital, Clinic of Pathology, Istanbul, Turkey
}

\section{Abstract}

Lymphoepithelial carcinoma (LEC) refers to a non-keratinized undifferentiated type of nasopharyngeal carcinoma. It rarely originates from the larynx mucosa. A 73-year-old male patient with a long term history of snoking was admitted to the hospital with a one-year history of dyspnea, dysphagia and dysphonia. Laryngeal LEC diagnosis was confirmed by a laryngeal biopsy. We presented a case report of locally advanced LEC of the larynx with only radiotherapy treatment.

Keywords: Lymphoepithelioma, larynx, radiotherapy

\section{INTRODUCTION}

Lymphoepithelial carcinoma (LEC) is a term used for neoplasms associated with non-keratinized undifferentiated nasopharyngeal carcinoma (WHO type 3). LEC is rarely caused by nasopharynx in the head and neck mucosa. It is characterized by the presence of lobules, sheets, nests and lymphoplasmacytic cell stroma which separates cords of neoplastic cells. It may also be associated with Epstein-Barr virus (EBV). Our report is a case of locally advanced LEC treated with radiotherapy.

\section{CASE PRESENTATION}

A 73-year-old male with a long term history of smoking applied with complaints of dysphagia, dysphonia and dyspnea for over a year, was admitted to our hospital with respiratory distress and hypoxia. Laryngoscopic examination of the patient revealed a supraglottic larynx mass.

It was a partially ulcerated lesion on the left aryepiglottic fold extending to the left ventricle (Figure 1a, b). The head and neck examinations revealed no palpable neck masses. Nasopharyngoscopy was used to detect the nasopharyngeal mass.
Tracheostomy was opened to the patient. Nasopharyngeal and laryngeal biopsy was performed. A computerized tomography scan of the neck revealed a supraglottic mass measuring $37 \times 30$ $\mathrm{mm}$ in size, extending over the vocal cord, aryepiglottic fold and piriform sinus (malignancy). The lesion invaded the arytenoid cartilage and extended into the paralaryngeal area and fat planes (Figure 2a, b).

Nasopharynx biopsy resulted in reactive lymphoid hyperplasia microscopic analysis, and immunohistochemistry of laryngeal biopsy confirmed the diagnosis of laryngeal LEC (Figure 3). There were significant nucleolus tumor cells with large vesicle nuclei (Figure 3a), and vesicular nucleoli with prominent nucleoli tumor cells between lymphoid cells (Figure 3b). The pan-cytokeratin staining (Figure 3c) was seen among tumor cells, CD3 positive lymphoid cells (Figure 3d) and CD20 positive lymphoid cells (Figure 3e). EBV RNA (EBER) was negative in the case. Radiotherapy was applied to our patient. The whole larynx area received 30 cures of 60 Gray radiotherapy. The tumor area was given 3 cures of 66 Gray radiotherapies. After 18 months of follow up recurrence or metastases was detected. The consent was obtained from patient. 


\section{DISCUSSION}

LEC is characterized by primitive, undifferentiated, nonkeratinized epithelial cells interpresed with non-neoplastic lymphocytes. LEC is reported in different parts of the head and neck mucosa like a sinonasal tract, salivary gland especially parotid gland, larynx and hypopharynx. LEC have been reported in the larynx rarely.

LEC constitutes $0.2 \%$ of all laryngeal cancers in men, mainly in elderly patients (1).
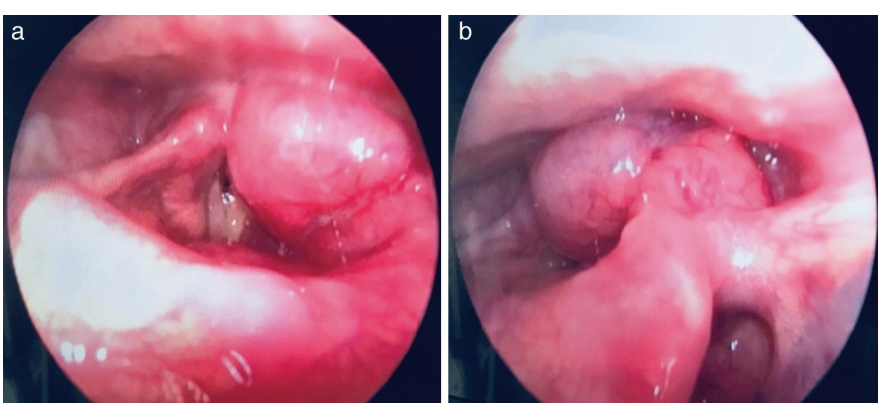

Figure 1a, b. Mass occupying supraglottic levels of the larynx. On endoscopic examination, a partially ulcerated lesion of the left aryepiglottic fold extending to the left ventricle
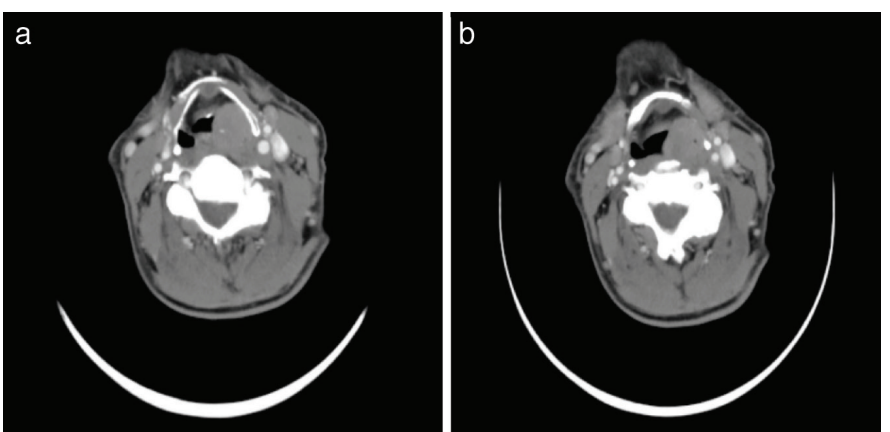

Figure $\mathbf{2 a}, \mathbf{b}$. Computed tomography scanning of the mass

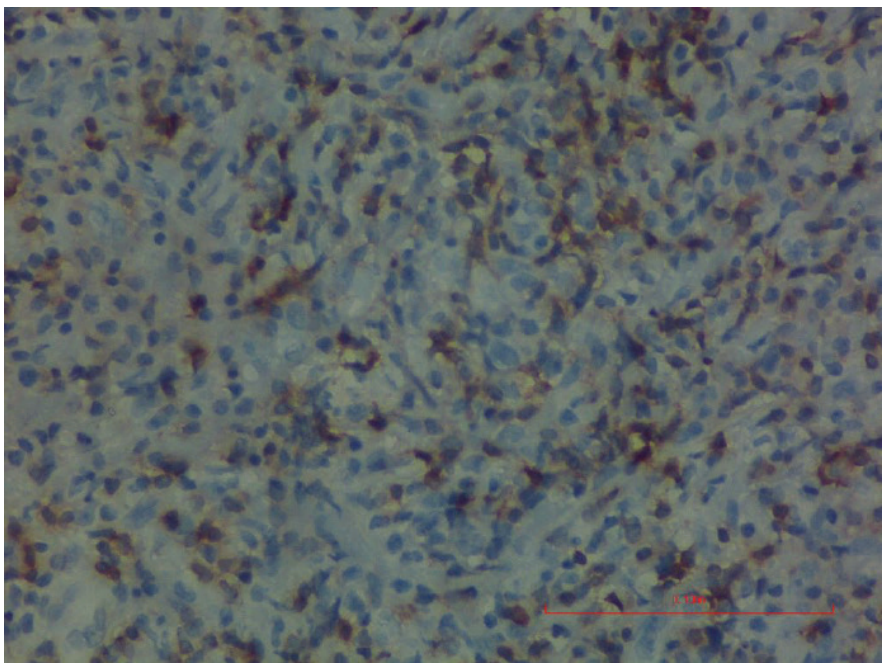

Figure 3a. Significant nucleolus tumor cells with large vesicle nuclei

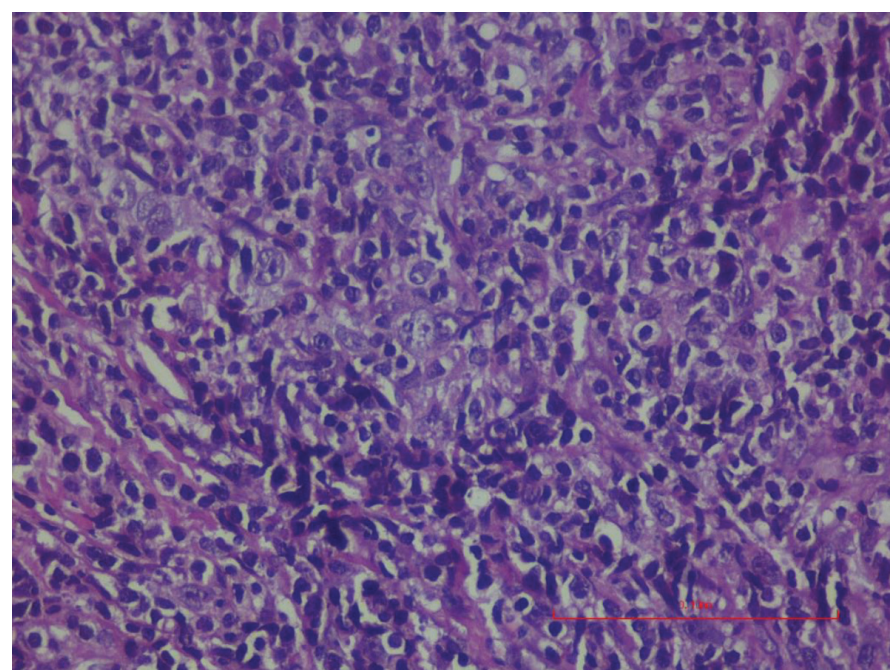

Figure $\mathbf{3 b}$. Between lymphoid cells, vesicular nucleoli with prominent nucleoli tumor cells

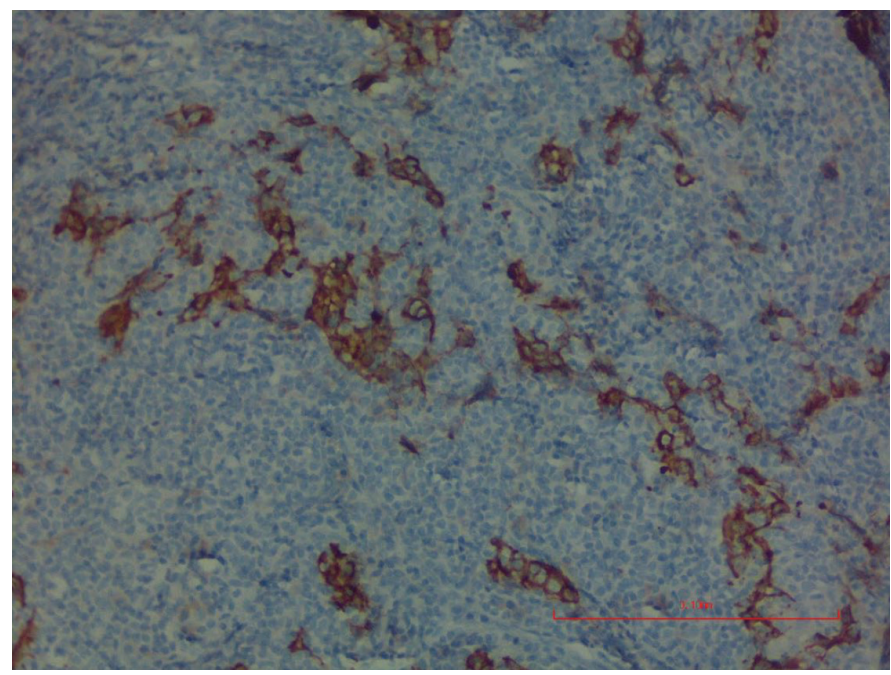

Figure 3c. Pan-cytokeratin staining

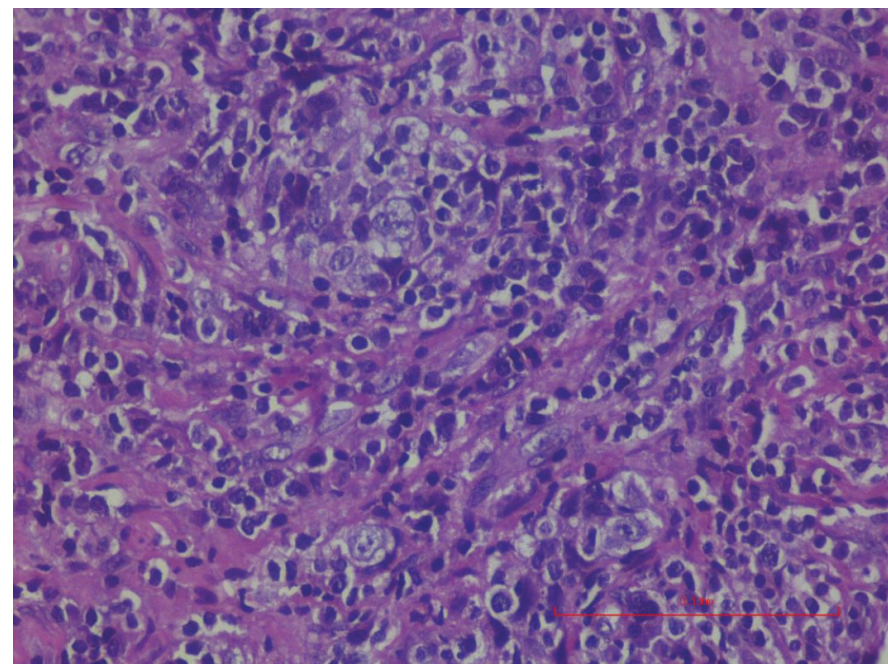

Figure 3d. Among tumor cells, CD3 positive lymphoid cells 


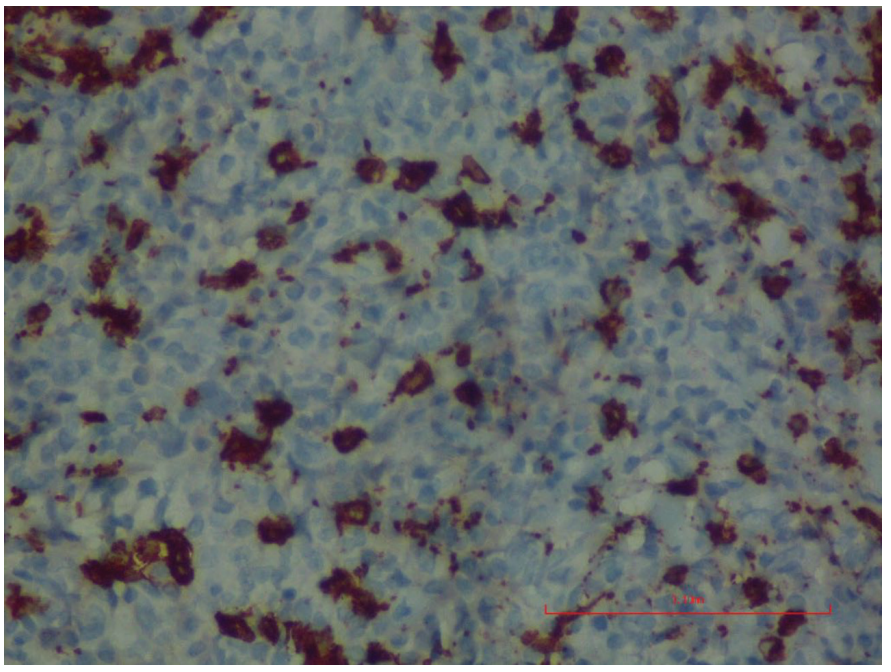

Figure 3e. Among tumor cells, CD20 positive lymphoid cells

Laryngeal LEC is considered to be similar to nasopharyngeal lenfoepithlioma except for its localization. Like nasopharyngeal carcinoma, LEC is also strongly associated with heavy cigarette smoking (2-5). In this case the patient also was a heavy smoker.

EBV antigens can be shown in immunohistochemical and molecular species. Macmillan et al. (5) reported no EBV involvement in their eight cases series but P53 expression was positive. Some authors declare that in endemic regions, EBER can be positive and can be used for diagnosis (5-7). Marioni et al. (8) published that EBV plays a limited role in the etiology of LEC of the larynx. In their study reviewed in the report of the 34 cases of LEC, only 16 were evaluated for the presence of EBV and it was demonstrated in only four cases (25\%). In our case, EBER was determined negative.

There is no special place for LEC but supraglottic localization is more prevalent (9), Micheau et al. (10). In a total of 1350 laryngeal surgical specimens, three are LEC cases in the larynx. Among the three cases, the LEC epiglottis was found on the laryngeal surface and was associated with the laryngocele in two cases. Toker and Peterson (11) reported a case of LEC of the larynx which had a peculiar property of tonsillar epithelium and might have been its origin. In our case, the lesion was in the supraglottic location and extended to the vocal band and ventricle. The mass was causing obstruction of the airway and emergent tracheostomy was performed. There was no case reported which required emergent tracheotomy in the literature.

Histopathologically, LEC is characterized by poorly differentiated, non-keratinized cells with large, round, vesicular nuclei, each containing a single large usually centrally located, basophilic to the deep red prominent nucleolus. Immunohistochemical staining is positive for keratin and epithelial membrane antigen. Differential diagnosis should be made by immunohistochemical methods in the positive cytokeratin menu for the identification of the malicious squamous component (4-6). Dysphonia, dysphagia, dyspnea and otalgia are the major symptoms. Lymphadenopathy can be encountered as well (2). In our case dysphonia and dyspnea were the main symptoms. There was no cervical lymph node encountered. Cervical lymph node metastasis was reported in the literature approximately $75 \%(5,7,8)$.

The distant distance metastasis rate is also high. Metastasis was not seen in this case (12).

Treatment of LEC is mostly radiotherapy or surgery with radiotherapy combination $(7,13)$. Laryngeal LEC is a highly radiosensitive disease and excellent local control rates can be achieved with radiotherapy (2). No comparative studies have been done, therefore, organ-preserving treatment methods are preferred. In our case, the patient received radioterapy after 18 months of follow up. No recurrence or metastases were detected.

\section{CONCLUSION}

Early detection is important and essential to distinguish the LEC from squamous cell carcinoma. The role of EBV is still not clear, and EBER can be used for differential diagnosis.

Radiotherapy which is the only treatment for local disease is recommended. Chemotherapy may also play a role in patients with advanced disease as well as in those with lymph node invasion.

\section{Ethics}

Informed Consent: The consent was obtained from patient.

Peer-review: Externally peer-reviewed.

\section{Authorship Contributions}

Concept: B.T., G.B., Y.U., Design: F.A., Y.U., Data Collection or Processing: Z.S., Analysis or Interpretation: B.T., P.Ö.N., Literature Search: F.A., Writing: B.T., I.A.A.

Conflict of Interest: No conflict of interest was declared by the authors.

Financial Disclosure: The authors declared that this study received no financial support.

\section{REFERENCES}

1. Singhi AD, Stelow EB, Mills SE, Westra WH. Lymphoepithelial-like carcinoma of the oropharynx: a morphologic variant of HPV-related head and neck carcinoma. Am J Surg Pathol 2010;34:800-5. 
2. Dubey P, Ha CS, Ang KK, El-Naggar AK, Knapp C, Byers RM, Morrison WH Nonnasopharyngeal lymphoepithelioma of the head and neck. Cancer 1998;82:1556-62.

3. Iezzoni JC, Gaffey MJ, Weiss LM. The role of Epstein-Barr virus in Iymphoepithelioma like carcinomas. Am J Clin Pathol 1995;103:308-15.

4. Wenig BM. Lymphoepithelial-like carcinomas of the head and neck. Semin Diagn Pathol 2015;32:74-86.

5. MacMillan C, Kapadia SB, Finkelstein SD, Nalesnik MA, Barnes L. Lymphoepithelial carcinoma of the larynx and hypopharynx: study of eight caseswith relationship to Epstein-Barr virus and p53 gene alterations, and review ofthe literature. Hum Pathol 1996;27:1172-9.

6. Singhi AD, Stelow EB, Mills SE, Westra WH. Lymphoepithelial-like carcinoma of the oropharynx: a morphologic variant of HPV-related head and neck carcinoma. Am J Surg Pathol 2010;34:800-5.

7. Narozny W, Betlejewski A, Stankiewicz C, Kamiński M. Ventriculosaccular lymphoepithelioma of the larynx: case report and literature review. Head Neck 1998;20:425-9.
8. Marioni G, Mariuzzi L, Gaio E, Portaleone S, Pertoldi B, Staffieri A. Lymphoeptihleiomal carcinoma of the larynx. Acta Otolaryngol 2002;122:429-34.

9. Dray T, Vargas H, Weidner N, Sofferman RA. Lymphoepitheliomas of the laryngohypopharynx. Am J Otolaryngol 1998;19:263-6.

10. Micheau C, Luboinski B, Schwaab G, Richard J, Cachin Y. Lymphoepitheliomas of the larynx (undifferentiated carcinoma of nasopharyngeal type). Clin Otolaryngol 1979;4:43-8.

11. Toker C, Peterson DW. Lymphoepithelioma of the vocal cord. Arch Otolaryngol 1978;104:161-2.

12. Tardío JC, Cristóbal E, Burgos F, Menárguez J. Absence of EBV genome inlymphoepithelioma-like carcinomas of the larynx. Histopathology 1997;30:126-8.

13. Coskun BU, Cinar U, Sener BM, Dadas B. Lymphoepithelial carcinoma of thelarynx. Auris Nasus Larynx 2005;32:189-93. 